\title{
A Blog-Centered IPTV Environment for Enhancing Contents Provision, Consumption, and Evolution
}

\author{
In-Young Ko, Sang-Ho Choi, and Han-Gyu Ko \\ Dept. of Computer Science, Korea Advanced Institute of Science and Technology, \\ 335 Gwahangno, Yuseong-gu, Daejeon, 305-701, Republic of Korea \\ \{iko, shchoi9, kohangyu\}@kaist.ac.kr
}

\begin{abstract}
There have been some efforts to take advantages of the Web for the IPTV domain to overcome its limitations. As users become the center of the contents creation and distribution, motivating user participation is the key to the success of the Web-based IPTV. In this paper, we propose a new IPTV framework, called a blog-centered IPTV, where personal blogs are the firstclass entities that represent user interests in IPTV contents. An IPTV blog provides a user with a set of interfaces for finding, accessing and organizing IPTV contents based on their needs, and becomes an active entity to join communities and to participate in making community contents evolved. We have implemented a prototype of the blog-centered IPTV, and showed that users can easily find and access their desired contents and successfully build community-based contents.
\end{abstract}

Keywords: IPTV, Web-based IPTV, Blog, Community, Social Networks.

\section{Introduction}

The Web is now being considered as an important platform for new IPTV (Internet Protocol Television). The essential characteristics of the Web such as openness, variety, and accessibility bring IPTV users with facilities to promote the content creation and distribution by allowing users to collaboratively participate in creating, organizing and sharing their contents [1]. There have been some efforts to take advantages of the Web for the IPTV domain such as Joost, Babelgum, and Metacafe ${ }^{1}$. To effectively motivate user participation, which is the key to the success of Webbased IPTV, more user-centric functions and facilities are needed than simply sharing of video contents [2].

In this paper, we propose a new IPTV framework, called a blog-centered IPTV, where personal Web logs (blogs) are the first-class entities that represent user interests in IPTV contents. An IPTV blog provides a user with a set of interfaces for finding, accessing and organizing IPTV contents based on their needs, and becomes an active entity to join communities and to participate in making community contents evolved. Although it has similar features with Blogosphere [3], as Fig. 1 depicts, the

1 http://www.joost.com/, http://www.babelgum.com/,

http://www.metacafe.com/

B. Benatallah et al. (Eds.): ICWE 2010, LNCS 6189, pp. 522 -526, 2010.

(c) Springer-Verlag Berlin Heidelberg 2010 
blog-centered IPTV covers the entire lifecycle of IPTV communities and supports user activities of content consumption, evolution, syndication, and provision. In addition, it automatically identifies potential IPTV communities by analyzing the social and personal characteristics of users, and recommends a user with an existing community to join or a potentially useful community to create [4].

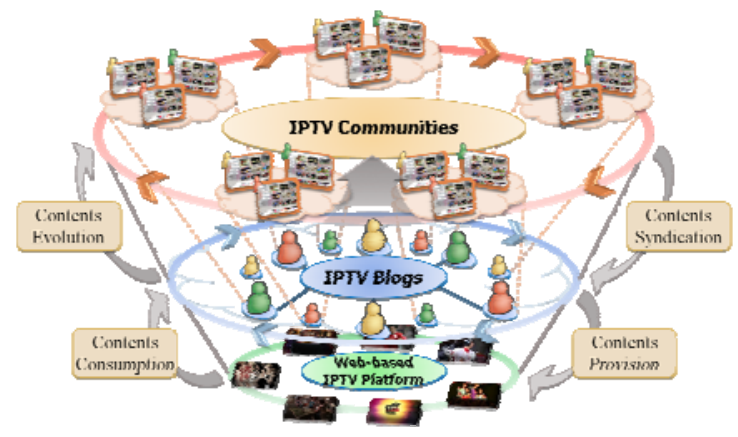

Fig. 1. Overview of the Blog-centered IPTV Environment

For an IPTV community, the IPTV blogs proactively contribute to accumulate and organize various contents that are relevant to the community. Community contents are then consumed by each blog in a personalized way. Users can easily locate and access the contents that meet their interests and needs via their IPTV blogs.

The rest of this paper is organized as follows. In Section 2, we explain the architecture of the blog-centered IPTV environment including essential components and their relations. Section 3 presents a blog-centered IPTV prototype and the paper ends in Section 4 with the conclusion and future work.

\section{Architecture of the Blog-Centered IPTV Environment}

As shown in Fig. 2, the architecture of the blog-Centered IPTV environment consists of three main layers: (a) Media Layer, (b) IPTV Blog Layer, and (c) IPTV Community Layer. The media layer stores and provides various resources including media contents and their metadata, and user-related information. The media contents are accessed via the media enabler that locates and streamlines the contents. The semantic enabler provides an ontology-based model and reasoning methods to represent and manage semantic metadata of IPTV contents. The social connector accesses user-related data and extracts social network information to be used for recommending potential blog communities.

In the IPTV blog layer, the IPTV blog handler provides a basic set of functions to create or customize a blog by using a template, to define the profile information of a blog, and to create channels that integrate and deliver blog contents to other users. The semantic tagging facilities allow users to put ontology-based semantic tags on multimedia contents. The semantic tags are automatically collected, monitored and analyzed to identify user preferences and to determine potential communities to be 
recommended to the users. The semantic media syndication component provides functions to enable blogs to subscribe for each others' contents based on their needs represented in an ontology-based semantic model. The contents mash-up engine supports users with a set of methods to integrate various types of contents such as texts, Web services as well as regular video contents, and make them delivered via the blog channels. The semantic search engine allows blogs to be equipped with semantically-based search capability to produce more relevant search results based on user preferences and interests.

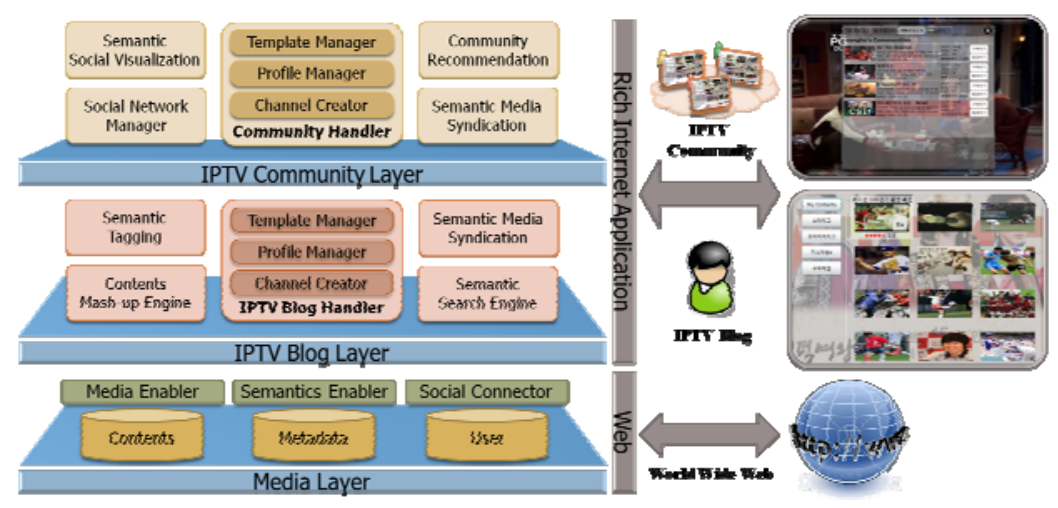

Fig. 2. Architecture of the blog-centered IPTV environment

Similar to the IPTV blog handler, the IPTV community handler at the IPTV community layer provides functionality of creating and managing IPTV communities. The social network manager identifies a social network of users centered on the owner of a blog based on their preferences and social relationship information. The semantic social visualization module visualizes a map of users in a social network and allows users to browse through the map based on user interests. The community recommendation module recommends users to create a new IPTV community or to join an existing one by comparing profiles of blogs and by grouping relevant blogs based on their semantic properties. A blog can subscribe for a community channels by using the functions provided by the semantic media syndication component.

\section{Prototype and Application}

To show the effectiveness of our blog-centered IPTV, we implemented a prototype. For IPTV clients, we used Silverlight ${ }^{2}$, which is one of the popular RIA (Rich Internet Application) technologies [5]. We used IIS (Internet Information Services) for the Web server and WMS (Windows Media Services) for the media server. The right side of Fig. 2 shows the screen shots of an IPTV blog and a community page. In a blog, metadata of relevant contents are arranged in a chronicle order with

${ }^{2}$ http: / /www. silverlight.net/ 
comments, rankings, tags and other annotations. An IPTV community page shows an aggregated view of information about the most relevant contents from the blogs in the community.

Users can find relevant contents through the semantic search capability, and as shown in Fig. 3 (left), the search result is displayed in a radar view, by which users can easily identify semantic closeness of the contents found toward their needs. Users can also browse through different categories of contents by centering a different content, and narrow down into a more specific set of contents by applying various semantic filters such as genres, time, location, emotions, etc. that are automatically enabled based on the context of the contents and users. By using the social network browser (the right-side screen shot in Fig. 3), users can navigate through a group of users who have similar interests, and access contents organized by other users. In addition, the users can create a new IPTV community by inviting other users in the social network.
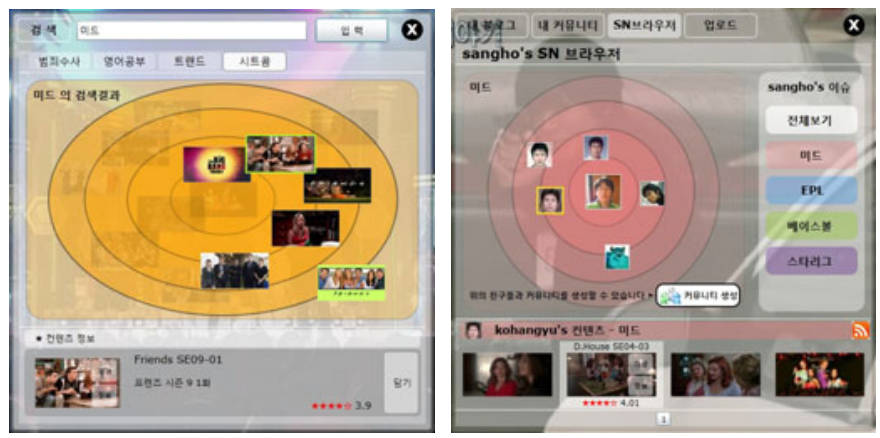

Fig. 3. Semantic search interface (left), and a social network browser (right)

\section{Conclusion}

In this paper, we proposed a new IPTV environment called a blog-centered IPTV, which provides facilities of semantically-based content search and visual browsing, and IPTV community recommendation and management. In this approach, personal blogs are the first class entities that organize IPTV contents in a personalized way, and actively find and join potential communities. We believe that this framework will enhance the provision, consumption, and evolution of IPTV contents by motivating more user participations. We are currently extending our approach by adding features to monitor community activities and to manage the lifecycle of communities according to them.

Acknowledgments. This work was supported by the IT R\&D program of MKE/ KEIT. [2008-S-006-02, Development of Open-IPTV (IPTV2.0) Technologies for Wired and Wireless Networks] 


\section{References}

1. O'Reilly, T.: What Is Web 2.0: Design Patterns and Business Models for the Next Generation of Software. Published on O'Reilly, Communications \& Strategies (1), 17 (First Quarter, 2007)

2. Koh, J., et al.: Encouraging participation in virtual communities. Communications of the ACM 50(2), 69-73 (2007)

3. Herring, S.C., Kouper, I., Paolillo, J.C., Scheidt, L.A., Tyworth, M., Welsch, P., Wright, E., $\mathrm{Yu}$, N.: Conversation in the Blogosphere: An Analysis From the Bottom Up. In: Proceedings of the 38th Annual Hawaii International Conference on System Sciences (HICSS'05), Big Island, Hawaii, January 3-6 (2005)

4. Ko, H.-G., Choi, S.-H., Ko, I.-Y.: A Community Recommendation Method based on Social Networks for Web 2.0-based IPTV. In: 16th International Conference on Digital Signal Processing, Santorini, Greece, July 5-7 (2009)

5. O’Rourke, C.: A Look at Rich Internet Applications. Oracle Magazine 18(4), 59-60 (2004) 